\title{
Normal Force Stabilizing Control Using Small EV Powered only by Electric Double Layer Capacitor
}

\author{
Kiyotaka Kawashima*, Toshiyuki Uchida*, Yoichi Hori**
}

This paper has two topics. The first one is development of novel electric vehicle (EV) powered only by "Electrical Double Layer Capacitor (EDLC)". The second one is normal force stabilizing (NFS) vehicle motion control using this experimental vehicle. The vehicle provides us useful experimental environment of EV's motion control, since EDLC power system can shorten charging time. Only 30 seconds-charging enables us 20 minutes driving experiments. The development of small EV and the vehicle control system are shown. Novel vehicle stabilizing control in rolling motion using differential torque is proposed. Normal force has strong connectivity with driving force and its sudden decrease causes tire slip, which makes the whole vehicle motion unstable. The simulation and experimental results are shown and the effectiveness of proposed method is discussed.

Keywords: Light Vehicles, On-Board Energy Storage System, Double Layer Capacitor, Vehicle Motion Control

\section{INTRODUCTION}

Electrical double layer capacitor (EDLC), whose energy density has drastically increased, is drawing much attention. EDLC has several advantages as following.

1. It can be charged and discharged very quickly without heat generation because it is not based on chemical reaction.

2. Capacitor's voltage level tells us the remaining energy level precisely.

3. Capacitor is very tough to endure the repetitions of charging and discharging.

4. Capacitor is environmental friendly because it does not use heavy metals.

Capacitors have been used as backup batteries of mobile PC's, printers, UPS's, etc. In the field of automobile, some fuel-cell and hybrid vehicles use capacitors not only for absorbing the regenerated energy but also for compensating the low efficiency of fuel-cell battery and secondary battery [1]. Long life duration of EDLC is also useful for starter battery of vehicles.

The purpose of making the capacitor vehicle is to develop the useful experimental environment of vehicle motion control taking the advantage of peculiar characteristic of large current charging. In the following section, the development of small EV "Capacitor

* Department of Electrical Engineering, the University of Tokyo, 7-3-1 Hongo Bunkyo Tokyo 113-8565, e-mail:kawashima@ horilab.iis.u-tokyo.ac.jp; uchida@horilab.iis.u-tokyo.ac.jp

** Institute of Industrial Science, the University of Tokyo, 4-6-1 Komaba Meguro Tokyo 153-8505, e-mail:hori@iis.u-tokyo.ac.jp
COMS (C-COMS; Fig. 1)" and the vehicle control system are described. In the third section, the vehicle normal force stabilizing (NFS) control method is also proposed. Normal force has strong connectivity with driving force and its sudden decrease causes tire slip, which makes the whole vehicle motion unstable. Basic simulation and experimental results of NFS control are given in the forth section.

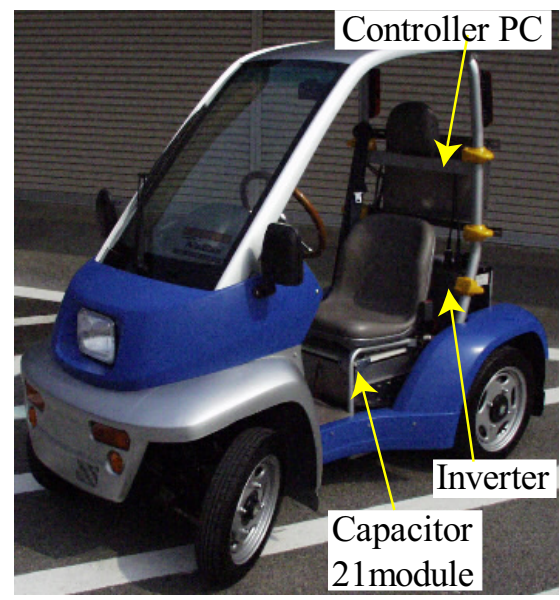

Fig. 1 Capacitor-COMS

\section{DEVELOPMENT OF SMALL ELECTRIC VEHICLE POWERED ONLY BY EDLC}

EDLC modules $(105 \mathrm{~V}, 85 \mathrm{~F})$ are installed on the vehicle.

\subsection{Merits of EDLC Application for EV}

EDLC application for EV has following advantages 
which secondary batteries do not have.

As EDLC is not based on chemical reaction and its internal resistance is small theoretically, large current charging is possible. EDLC is also more than 10 times as durable as for repetition of charging and discharging. In addition, EDLC voltage level tells us remaining energy level very precisely against that estimation of energy level on secondary battery is very difficult. Only 30 seconds-charging realizes 20 minutes driving in our system.

We adopted the system that EDLC is directly connected to inverter. The advantages of this system are effective space utilization and high energy efficiency compared to utilizing DC-DC converter. Fig. 2 shows voltage and current data of driving experiment. The figures show that increase of the current compensates decrease of the voltage in the low voltage region. Minus current indicates regenerative current.
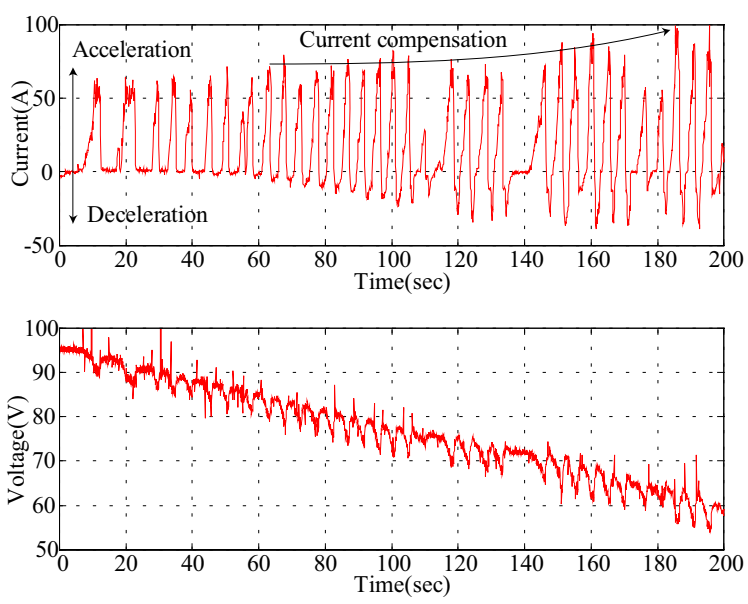

Fig. 2 Driving experimental results

Table 1 shows the comparison of EDLC parameters with other secondary batteries [2][3].

Table 1 Comparison with other secondary batteries

\begin{tabular}{|c|c|c|c|c|}
\hline & \multicolumn{2}{|c|}{ Energy density } & Power density & Cycle \\
\hline & $\mathrm{Wh} / \mathrm{kg}$ & $\mathrm{Wh} / 1$ & $\mathrm{~W} / \mathrm{kg}$ & \\
\hline Lead & 35 & 80 & 200 & 500 \\
\hline NiMH & 65 & 155 & 200 & 1000 \\
\hline Li-ion & 110 & 160 & 200 & 1000 \\
\hline EDLC* & 5.1 & 6.2 & 2420 & 100,000 \\
\hline
\end{tabular}

*Nisshinbo $3 \mathrm{~V}$ cell

Although the EDLC's energy density is very small, the power density is more than 20 times as large as other devices and cycle life is more than 10 times. These characteristics enable fast charging and make an EV suitable for vehicle's experiment, where lots of experiments are needed in the same condition in short experimental time.

This system can be applied to AGV (Automatic Guide Vehicle) or AGF (Automatic Guide Forklift) whose trajectory is fixed and required repetitive short-term use.

\subsection{EDLC Specification}

Table 2 shows the specification of EDLC module installed in C-COMS. A Module has 5 cells and maximum voltage is $3.0 \mathrm{~V}$. 21 EDLC modules $(7$ series $\times$ $3)$ are installed on the vehicle.

Table 2 Specification of EDLC module

\begin{tabular}{|c|c|}
\hline Voltage & $15 \mathrm{~V}$ \\
\hline Capacitance & $200 \mathrm{~F}$ \\
\hline Energy density & $3.4 \mathrm{Wh} / \mathrm{kg}$ \\
\hline Power density & $4.0 \mathrm{~W} / \mathrm{kg}$ \\
\hline Internal resistance & $8.9 \mathrm{~m} \Omega$ \\
\hline Mass & $1.9 \mathrm{~kg}$ \\
\hline Volume & $155 \mathrm{mmx} 66 \mathrm{mmx} 154 \mathrm{~mm}$ \\
\hline Number of modules & 21 \\
\hline
\end{tabular}

\subsection{Vehicle Specification}

Our small vehicle named C-COMS is a one-seater vehicle with two in-wheel motors. Drive train consists of two inverters and two permanent magnet synchronous motors. Table 3 shows its details.

The original inverter could not give torque command to each motor independently and it has about $300 \mathrm{msec}$ time lag from the acceleration command to the motor. Therefore we designed a new inverter which can generate reference torque independently and has little time lag to realize novel motion control of vehicle dynamics. This inverter was developed with due consideration for the voltage change.

Table 3 Drive train of C-COMS

\begin{tabular}{|c|c|}
\hline \multicolumn{2}{|c|}{ Motor } \\
\hline Category & PM SM \\
\hline Phase/Pole & $3 / 12$ \\
\hline Rating Power/Max & $0.29 \mathrm{~kW} / 2 \mathrm{~kW}$ \\
\hline Max torque & $100 \mathrm{Nm}$ \\
\hline Max speed & $50 \mathrm{~km} / \mathrm{h}$ \\
\hline \multicolumn{2}{|c|}{ Inverter } \\
\hline \hline Control method & PWM vector control \\
\hline DC input & $30 \mathrm{~V}-100 \mathrm{~V}$ \\
\hline SW frequency & $20 \mathrm{kHz}$ \\
\hline
\end{tabular}

\subsection{Development of Capacitor-COMS}

Fig. 3 shows the vehicle control system.

We developed following four new components which the original EV did not have.

- ECU (Electrical Control Unit) for generating reference torques and storing experimental data

- EDLC box and newly designed inverter

- Speed detector using PIC (Peripheral Interface Controller)

- Steering encoder, acceleration/gyro sensor 
ECU was developed using PC104 standard embedded PC module. The merit of using this standard is that this $\mathrm{PC}$ is very small and has high extendibility. ECU consists of CPU boards, DC/DC converter, 2.5 inch hard disk drive, and a cooling fan. AD board reads the acceleration command and ECU calculates the required torque command. Sensors' information and state variables are logged and stored in the HDD.

Speed detector from the magnet sensor pulses was developed using micro computers. In-wheel motors have magnet speed detector. Three pulses are output together with 120 (deg) phase lag each. We used PIC (8 pin DIP), which is very small micro computer and inexpensive, for converting three phase pulses of magnet sensors to speed pulse and rotational direction signal.

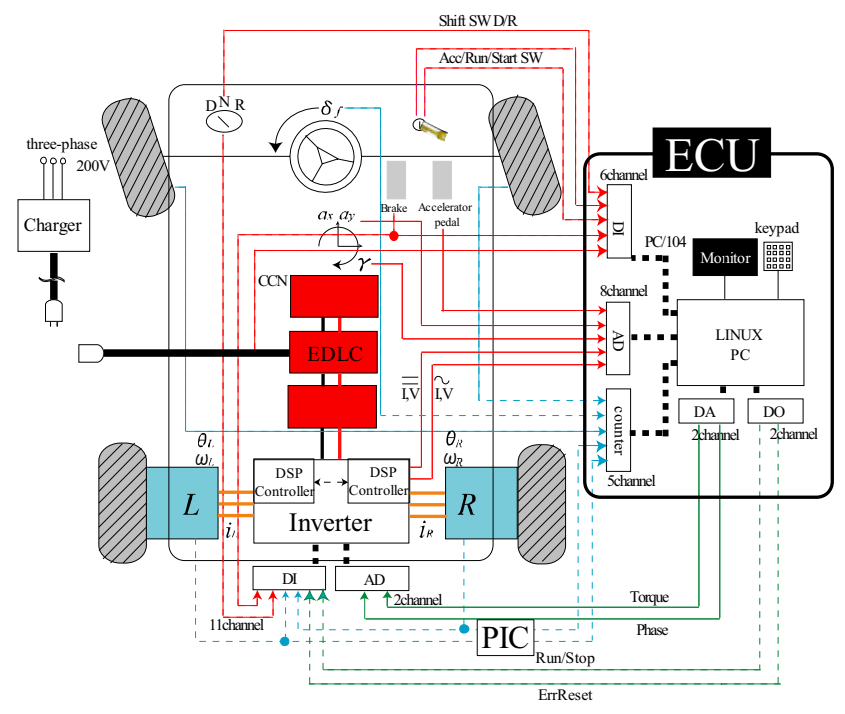

Fig. 3 Vehicle control system

\section{NORMAL FORCE STABILIZING CONTROL USING ACCELERATION INFORMATION}

\subsection{Motion Control on Electric Vehicle}

As we have pointed out, EV has the following four remarkable advantages [4] [5].

1. Motor's torque generation is $10-100$ times faster than engine (Motor can be controlled less than $1 \mathrm{msec})$. This advantage enables us to realize high performance adhesion control, skid prevention and slip ratio control.

2. Motor's torque can be known easily by observing the motor current. This property can be used for road condition estimation.

3. As a motor is compact and not so expensive if it is divided into four, it can be equipped for each wheel. This realizes high performance on vehicle motion control.

4. There is no difference between acceleration and deceleration control. Just giving minus torque command, vehicle can be decelerated.
Utilizing these advantages slip prevention control, road condition estimation, yaw rate control and other vehicle stabilizing control methods had been proposed. For example, control with independent wheel torque control [6], yaw-moment stabilizing control using yaw-moment observer [7] had been studied. Self-aligning torque estimation [8] and cornering stiffness estimation [9] are also important research subjects.

\subsection{Normal Force Stabilizing Control}

We will show that normal force on each tire can be calculated by using acceleration information. Considering of moment balance, displacement of hypothetical center of gravity (HCG) can be estimated by these normal forces. Here, hypothetical means that suspension system is not taken into account.

\subsubsection{Difference with Other Conventional Rolling Control Method with Active Suspension System}

Though active rolling controls using active suspension system are widely studied [10]-[12], we propose the novel rolling control method utilizing differential torque on each tire.

We focus on that normal force on each tire is calculated using acceleration information and it is possible to estimate displacement of $\mathrm{HCG}$. The purpose of NFS is to control displacement of HCG with differential torque. We do not need extra space for mounting active suspension system and we can utilize the algorithm easily by updating the software.

\subsubsection{Normal Force Calculation on Each Tire}

Considering of moment balance, the normal forces on the center of front and rear shaft at acceleration (Fig. 4, 5) are given as

$$
\begin{aligned}
& F_{z-f}=\frac{M}{l_{f}+l_{r}}\left(l_{r} g-h a_{x}\right) \\
& F_{z-r}=\frac{M}{l_{f}+l_{r}}\left(l_{f} g+h a_{x}\right) .
\end{aligned}
$$

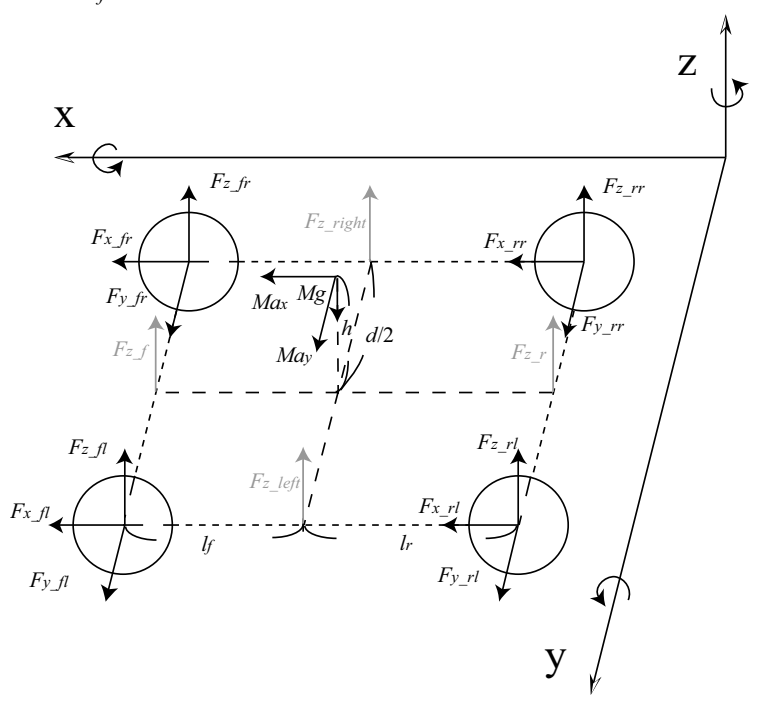

Fig. 4 Normal force on each tire 
Where $\mathrm{M}$ is vehicle weight, $\mathrm{g}$ acceleration of gravity, $a_{x}$ and $a_{y}$ vehicle longitudinal and lateral acceleration, $\mathrm{h}$ height of HCG from ground, $l_{f}$ and $l_{r}$ distance between HCG and front and rear shaft, d right and left tire distance, $\delta_{f}$ steering angle.

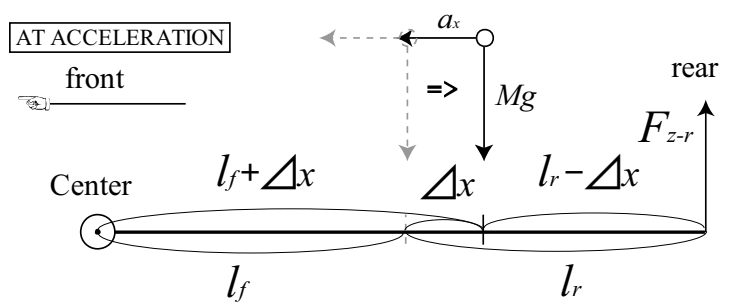

Fig. 5 Moment balance of vehicle

Normal force on each tire is calculated as following Eqs. (3-a,b,c,d) [13].

$$
\begin{aligned}
& F_{z-f r}=\frac{1}{2} \frac{M}{l_{f}+l_{r}}\left(l_{r} g-h a_{x}\right)+\frac{h M a_{y}}{d} \\
& F_{z-f l}=\frac{1}{2} \frac{M}{l_{f}+l_{r}}\left(l_{r} g-h a_{x}\right)-\frac{h M a_{y}}{d} \\
& F_{z-r r}=\frac{1}{2} \frac{M}{l_{f}+l_{r}}\left(l_{r} g+h a_{x}\right)+\frac{h M a_{y}}{d} \\
& F_{z-r l}=\frac{1}{2} \frac{M}{l_{f}+l_{r}}\left(l_{r} g+h a_{x}\right)-\frac{h M a_{y}}{d}
\end{aligned}
$$$$
(3-a, b, c, d)
$$

\subsubsection{HCG Calculation}

Defining $\Delta x, \Delta y$ as displacement of HCG (normal force instability indices), the momentum balance equation in longitudinal direction is expressed by Eq. (4).

$$
-F_{z-f}\left(l_{f}+\Delta x\right)+F_{z-r}\left(l_{r}-\Delta x\right)=0
$$

$\Delta x$ is given as following equation.

$$
\begin{aligned}
\Delta x & =\frac{-F_{z-f} l_{f}+F_{z-r} l_{r}}{F_{z-f}+F_{z-r}} \\
& =\frac{a_{x}}{g} h
\end{aligned}
$$

In a similar way, $\Delta y$ is given as

$$
\Delta y=\frac{a_{y}}{g} h .
$$

Eqs. (5) and (6) indicate that indices $\Delta x$ and $\Delta y$ are proportional to acceleration. To suppress $\Delta y$, differential torques are commanded on each motor. Fig. 6 shows the block diagram of this control method.

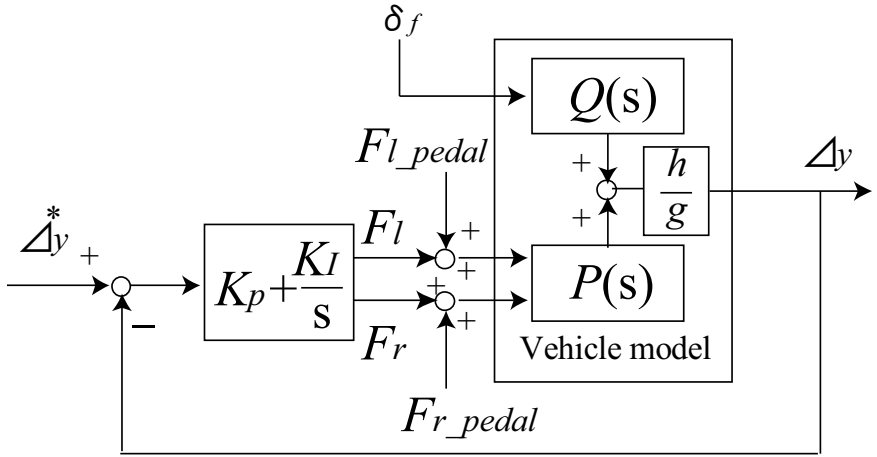

Fig. 6 NFS control diagram

\subsection{Simulation Results of NFS Control}

The simulation results in right turning and sinusoidal steering input are shown in Fig. 7. In actual experiment, acceleration information contains much noise, we need to eliminate noise with low pass filter.

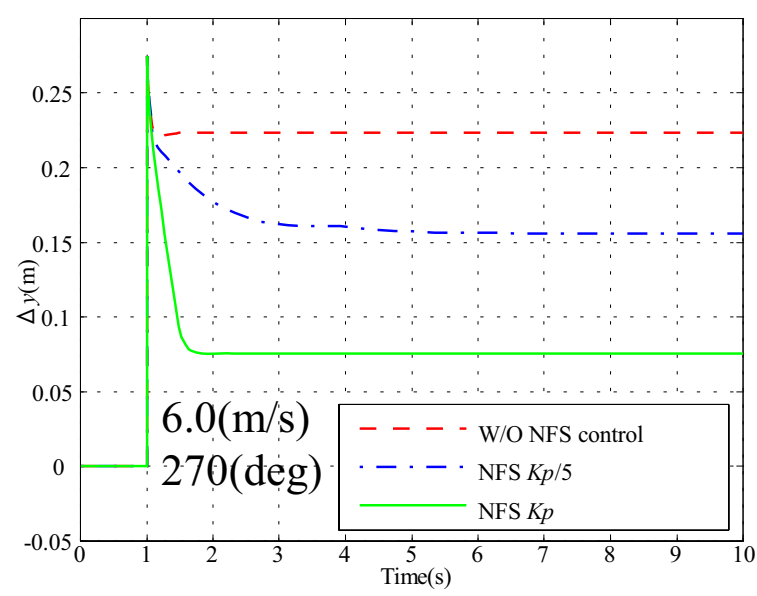

(a) Step steering input

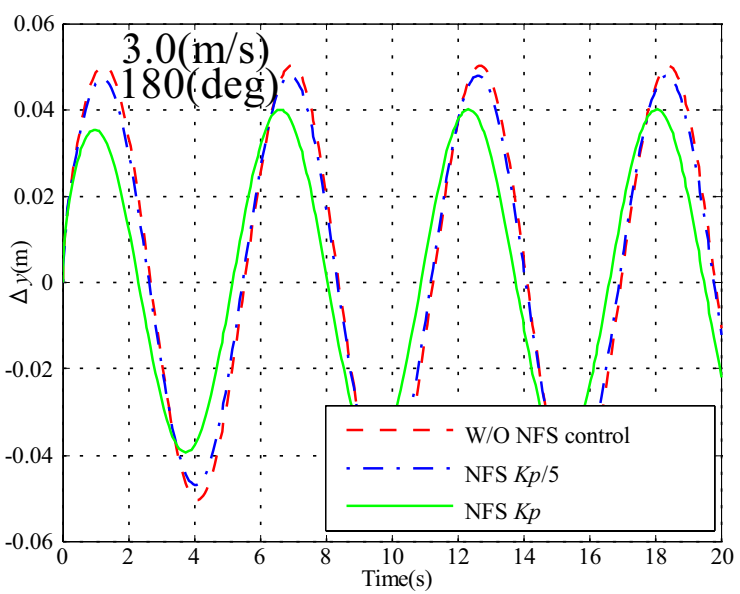

(b)Sinusoidal steering input

Fig. 7 Simulation results of NFS control

In the simulation, the reference variable $\Delta y^{*}$ is designed as zero. It is impossible to suppress $\Delta y$ to zero because of torque saturation. But these simulation 
results show the effectiveness of NFS control.

\section{EXPERIMENTAL VERIFICATION OF NFS CONTROL}

\subsection{Experimental Condition}

Experimental conditions is that driver turns the steering wheel as step and sinusoidal under constant velocity. Experimental terms are followings,

- Step steering input, $\Delta y^{*}$ is zero

- Sinusoidal steering input, $\Delta y^{*}$ is zero

- Step steering input, $\Delta y^{*}$ is generated from steering angle

- Sinusoidal steering input, $\Delta y^{*}$ is generated from steering angle

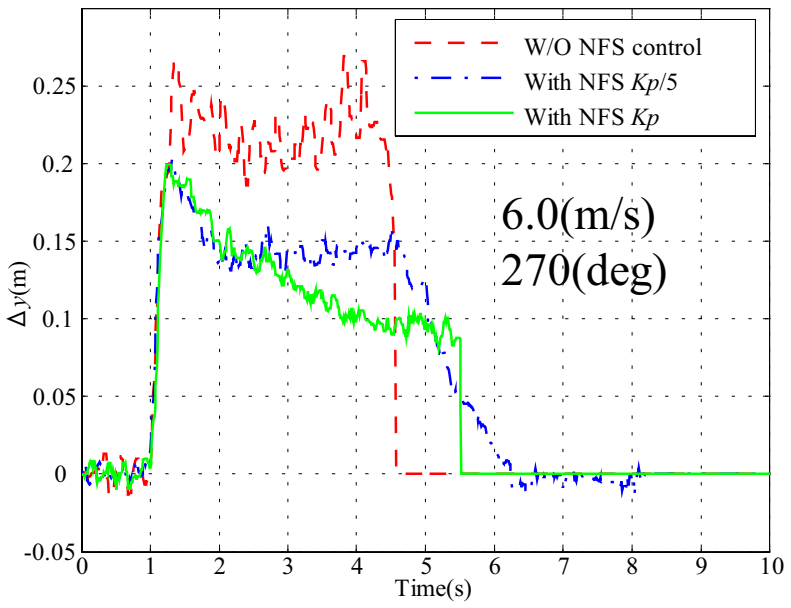

(a)Step steering input

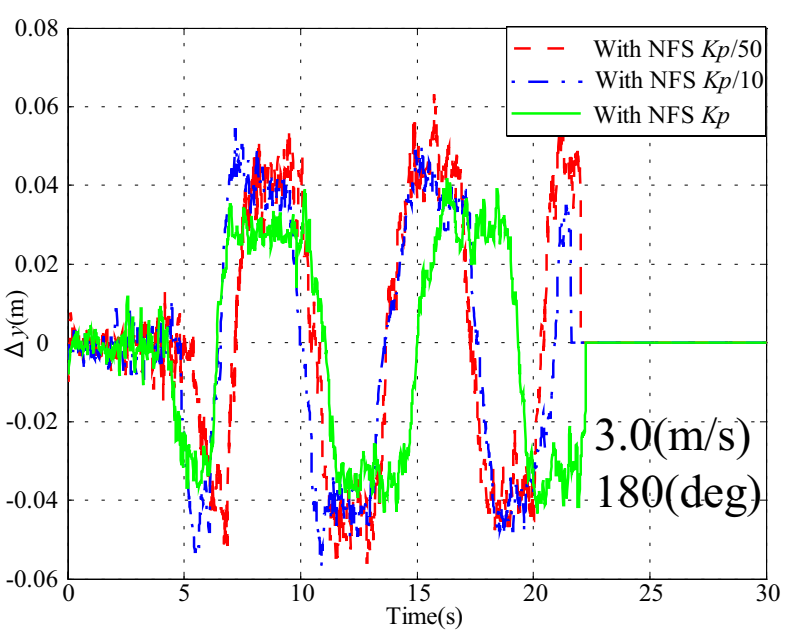

(b) Sinusoidal steering input

Fig. 8 Experimental results of NFS control when $\Delta y^{*}$ is zero

\subsection{Experimental Results of NFS Control}

In this section, four kinds of experimental results are shown mentioned above.

\subsubsection{Experimental Results of NFS Control when $\Delta y^{*}$ is Zero}

To know the basic vehicle behavior with differential torques, NFS control experiment is applied when $\Delta y^{*}$ is zero. The experimental results of step and sinusoidal steering input are shown in Fig. 8. With the larger control gain $K p$, the differential torques on right and left tires suppress $\Delta y$ more effectively. These figures correspond to the simulation results in the previous section.

4.2.2 Following Capability of $\Delta y$ to $\Delta y^{*}$

Fig. 9 are the cases that $\Delta y^{*}$ is generated as

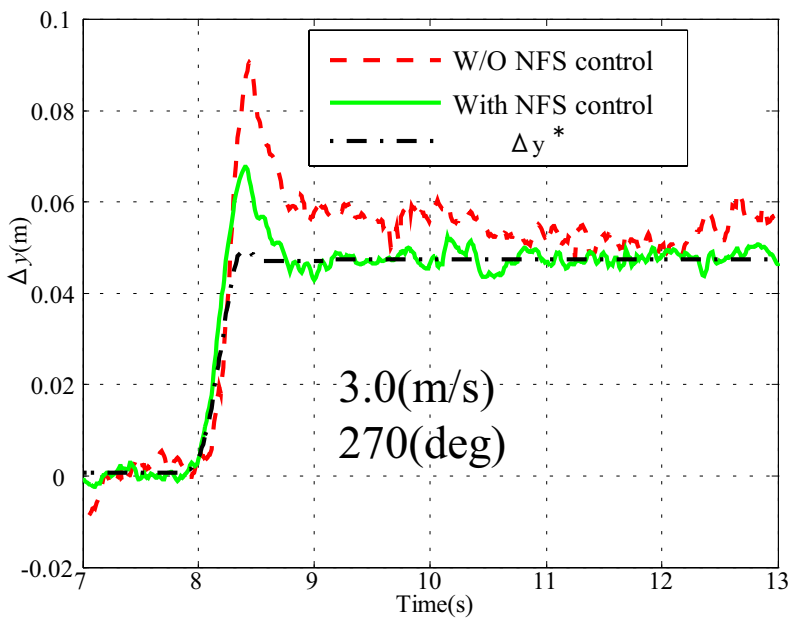

(a) Step steering input

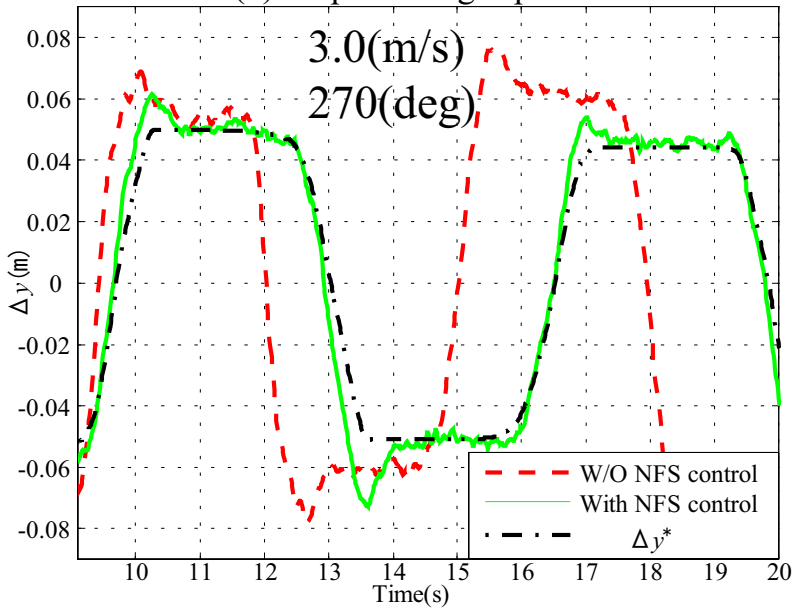

(b) Sinusoidal steering input

Fig. 9 Experimental results when $\Delta y^{*}$ is generated from steering angle and velocity

following Eq. (7) using gain $K$, vehicle's velocity $V$.

$$
\Delta y^{*}=K \cdot V \cdot \delta_{f}
$$

Fine $\Delta y$ suppression and following capability to $\Delta y^{*}$ is verified with these results. Consequently, we can control and design the displacement of HCG in lateral direction, which is one of vehicle turning characteristics, by the differential torques of right and left motors. It is very important to control the displacement of HCG because it is related to not only 
"ride quality", but also "vehicle active safety" in turning motion. Normal force has strong connectivity with driving force whose sudden decease would make vehicle motion unstable. NFS control method will be one of the solutions of vehicle safety and ride quality in turning motion.

\section{CONCLUSION}

In this paper, the development of experimental vehicle powered only by EDLC is introduced and novel vehicle stabilizing control method using lateral acceleration information is proposed. Short charging time realized by EDLC power system provides us useful experimental environment. The simulation and experimental results indicate the effectiveness of NFS control method. We do not need extra space for any hardware and we can utilize the algorithm only changing the software. Dangerous steering input is effectively suppressed and $\Delta y$ is controlled with reference variables. By controlling $\Delta y$, the novel vehicle active safety is realized in turning motion.

\section{REFERENCES}

[1] Michio Okamura, Electrical double layered capacitor and storage system (in Japanese), pp.25-31, Nikkan-KogyoShimbunsya

[2] Shintaro Azumi, "Investigative research of Life Cycle Assessment on electric vehicle (in Japanese)", Tottori University of Environmental Studies, pp.10, February 2005

[3] http://www.jari.or.jp/ja/denki/denki.html (in Japanese), Japan Automobile Research Institute

[4] Yoichi Hori, "Future Vehicle Driven by Electricity and Control-Research on Four Wheel Motored "UOT Electric March II", IEEE Transaction on Industrial Electronics, Vol.51, No.5, October 2004

[5] Shinichiro Sakai et al., "Novel skid detection method without vehicle chassis speed for electric vehicle, JSAE Review (Elsevier Science)", Vol.21, No.4, pp.504, 2000

[6] Motoki Shino, Masao Nagai, "Independent wheel torque control of small-scale electric vehicle for handling and stability improvement", JSAE Review, Vol.24, pp.449-456, 2003

[7] Hiroshi Fujimoto, Takeo Saito, Toshihiko Noguchi, "Yaw moment stabilizing control of small electric vehicle", AMC2004-Kawasaki, pp.35-40, 2004

[8] Daisuke Sekiguchi, Toshiyuki Murakami, "Vehicle steering assist by estimated self aligning torque in skid condition", AMC2004-Kawasaki, pp.269-273, 2004

[9] Akio Tsumasaka, Hiroshi Fujimoto, Toshiyuki Noguchi, "Cornering stiffness estimation of electric vehicle based on yaw moment observer (in Japanese)", National Convention Record IEE of Japan -Industry Applications Society, II pp.551-552, 2003

[10] http://ford.com/en/innovation/safety/accidentAvoidance/ rollStabilityControl.htm, Ford Motor Company

[11] David J.M. Sampson, David Cebon, "Active Roll Control of Single Unit Heavy Road Vehicles", Vehicle System
Dynamics, Vol.40 No.4 pp.229-270, 2003

[12] Antony Snell, "An Active Roll-Moment Control Strategy for Narrow Tilting Commuter Vehicles", Vehicle System Dynamics, Vol.29 No.5 pp.277-307, 1998

[13] Masato Abe, Vehicle dynamics and control (in Japanese), Sankaido, pp.150-152, 1992

\section{BIOGRAPHIES}

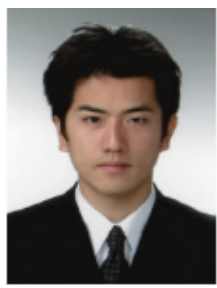

Kiyotaka Kawashima received the M.S. degree in Electrical Engineering from the University of Tokyo in 2006, and proceeded to doctoral course in the University of Tokyo. He is a research assistant of the department. His research field is motion control of electric vehicle and estimating the ELDC as the power source of electric vehicle.

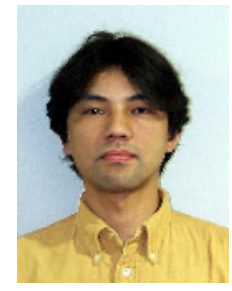

Toshiyuki Uchida is working as an official engineer in Department of Electrical Engineering the University of Tokyo.

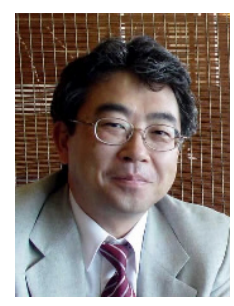

Yoichi Hori received PhD degrees in Electrical Engineering from the University of Tokyo in 1983 and joined the Department of Electrical Engineering as a Research Associate. He later became a Professor in 2000. In 2002, he moved to the Institute of Industrial Science as a Professor of Information \& Electronics Division. His research fields are control theory and its industrial application to motion control, mechatronics, robotics, electric vehicle, etc. He worked as Treasurer of IEEE Japan Council and Tokyo Section during 2001-2002. He is now an AdCom member of IEEE-IES. He was the Vice President of IEE-Japan IAS in 2004-2005. He has been the chairman of ECaSS Forum since 2005. $\mathrm{He}$ is the program chairperson of EVS-22. IEEE Fellow. 